\title{
TRANSUMBLICAL VERSUS INFRAUMBLICAL PNEUMOPERITONEUM: A COMPARATIVE STUDY.
}

1. MBBS, FCPS (Surgery) Ex. Associate Professor Sargodha Medical College/ DHQ Teaching Hospital Sargodha.

2. MBBS, FCPS (Anaesthesia) Assistant Professor DHQ Teaching Hospital Sargodha.

3. MBBS, FCPS (Medicine) Assistant Professor Sheikh Zayed Hospital, Lahore.

4. MBBS, FCPS (Surgery) Associate Professor DHQ Teaching Hospital Sargodha.

5. MBBS, FCPS (Surgery)

Senior Registrar

DHQ Teaching Hospital Sargodha.

6. MBBS, FCPS (Surgery)

Assistant Professor

DHQ Teaching Hospital Sargodha.

Correspondence Address:

Dr. Waseem Sadiq Awan

House No. E 15/F Lane No. 1 Street

No.7,

Officers Colony, Cavalry Ground

Lahore.

drwasimawan@hotmail.com

Article received on:

07/10/2019

Accepted for publication:

$15 / 12 / 2019$
Waseem Sadiq Awan', Raza Farrukh², Umme Bilqees ${ }^{3}$, Ahmad Hassan Khan ${ }^{4}$, Ahmad Aziz Jillani ${ }^{5}$, Khalid Mahmood ${ }^{6}$

ABSTRACT... Objectives: In Laparoscopic surgery placement of primary port is an important step and is often associated with complications. Our objective is to compare the merits and demerits like safety, efficacy of the site, time of entry of the port, per-operative and post-operative complications of infrumbilical (IU) with transumbilical (TU) approach for placement of this port. So on the basis of best clinical evidence get a clinical direction for a better site for placement of primary port. Study Design: Prospective randomized controlled trial. Setting: Surgical unit II of DHQ Hospital/ Sargodha Medical College (University of Sargodha) Sargodha. Period: June 2015 to December 2017. Material \& Methods: 238 Patients aged 16-70 years, fit for surgery, under general anesthesia were included in this study after informed consent. Patients who were unfit for general anaesthesia, who were Imuno-compromised, diabetics, CLD, with ascites, patients with suspected malignancy, with previous surgery in the umbilical region like midline laparotomy scar, previous hernia repair in this region were excluded from this study. Permission was sought from and granted by institutional ethical committee. Results: A total number of 238 patients were included. Group A (Trans-Umbilical) and Group B (Infra-Umbilical) contained 119 cases each. There were 147 female and 91 male. (M: F ratio of 1: 1.615). Conclusion: Based on results from this study and previous literature, it may be concluded that Transumbilical (TU) placement of primary port for creation of pneumoperitoneum is superior to infrumbilical (IU) approach.

Key words: Laparoscopic Surgery, Primary Port, Port Site Complications, Pneumoperitoneum, Safety Measures for Port Placement.

Article Citation: Awan WS, Farrukh R, Bilqees U, Khan AH, Jillani AA, Mahmood $K$. Transumblical versus Infraumblical pneumoperitoneum: A comparative study. Professional Med J 2020; $27(2): 388-394$.

DOI: 10.29309/TPMJ/2020.27.2.4224

\section{INTRODUCTION}

Laparoscopic surgery is now a time tested, recognized and well-established way of treating different abdominal and pelvic pathologies. The introduction of Laparoscopic surgery has revolutionized the field of abdominal and pelvic surgery, since its inception about three decade back by a French surgeon Mauret in 1987..$^{1,2}$

Pneumoperitoneum is to elevate the anterior abdominal wall and to make it more spacious, for the safe manipulation of different laparoscopic instruments in the abdominal cavity. ${ }^{3}$ Gases used are carbon dioxide, Nitrous oxide and Helium. Creating pneumoperitoneum by the placement of the primary port is very important as about half of the complications in routine laparoscopic surgery are related to this step. ${ }^{4}$
Routinely there are two sites for the placement of primary trocar to create pneumoperitoneum. More commonly used site is the infraumbilic and second is the transumbilic route. Even today none of the available methods of entry into the peritoneal cavity for creation of pneumoperitoneum are free of complications. ${ }^{5}$

\section{MATERIAL \& METHODS}

It is a prospective randomized controlled trial conducted from June 2012 to Jan 2018, at DHQ Hospital/ Sargodha Medical College (University of Sargodha). Non-probability consecutive sampling technique was used. The patients aged 16-70 years, fit for surgery, undergoing laparoscopic surgeries under general anesthesia were included in this study after informed consent. Patients who were unfit for general anaesthesia, 
who were Imuno-compromised, diabetics, CLD, with ascities, patients with suspected malignancy, with previous surgery in the umbilical region like midline laparotomy scar, previous hernia repair in this region were excluded from this study.

A total number of 238 patients at Unit II of department of General Surgery who fulfilled inclusion criteria were included in this study. Patients were allocated randomly into two groups by lottery method; Group A (Trans-Umbilical) and Group B (Infra-Umbilical) 119 and 119 respectively. Preoperative, intraoperative and postoperative data was collected. A written informed consent was obtained from patients to be included in the study. The data was collected on pre-designed printed proforma. This included the personal details of the patient e.g. Name, age, sex, history of presenting complaints, history of previous abdominal surgery, abdominal examination, BMI for obesity and concomitant diseases (diabetes, hypertension, bleeding disorder and any cardiac and respiratory issue).

Patients were followed on $3^{\text {rd }} 7^{\text {th }}$ and $10^{\text {th }}$ post discharge day and then after one month, three months and one year after surgery. The outcome variables taken into account were the trocar placement time, complications like hematoma formation, ecchymosis, subcutaneous emphysema, port site infection and postoperative hernia formation. The data was entered into SPSS and analyzed though its statistical package.

\section{Pre-operative Preparation}

All the patients were advised to take bath in the morning of surgery. The male patients were advised to get the abdominal hair clean, umbilicus to wash meticulously with anti-septic soap and to remove any debris deep in the umbilicus. Prophylactic antibiotics, $1 \mathrm{G}$ Cephalosporin was given in the pre anesthesia room about half an hour before the induction of anesthesia. All SOPs were adopted to control and prevent infection like adoption of standardized sterilization methods and all safety measures during placement and removal of trocars were observed. The instruments were sterilized as per protocol; dismantled and thoroughly washed with running tap water, all blood and debris were removed and put in glutaraldehyde for minimally half an hour. The antiseptic solution was changed after every case and minimal immersion time was half an hour. The procedure was done under general anesthesia.

\section{Operative Technique}

Our aim is to analyze the best way of pneumoperitonium and the goal is to have a safe and complication free pneumoperitoneum. The umbilicus is a natural scar, a structure deeper than the adjacent anterior abdominal wall where Peritoneum is fixed, wall is thin and less vascular.

\section{Infraumbilical port (IU)}

In primary trocar, the aim is to avoid iatrogenic injuries. We used the Hasson's technique. Hasson introduced this method in $1971 .{ }^{3} \mathrm{~A}$ small i.e., 1 to $2 \mathrm{~cm}$ incision is made in the infra umbilical area. All layers of anterior abdominal wall are dissected, peritoneum opened and trocar placed to be attached with gas nozzle to create pneumoperitoneum. ${ }^{6}$ Sometimes there was leakage of air around the trocar and the gauze pieces were packed in the wound and even occasionally towel clips had to be applied. Although this is the most common site for the placement of primary port however, likely drawback of this incision is more postoperative bad scar and umbilical deformity.

\section{Transumbilical port (TU)}

Umbilicus is a cone shaped structure with its apex towards surface, the skin fascia and peritoneum are fused and adherent. There is no fat and preperitoneal layer, so the umbilicus is thin even in obese patients and this allow an easy access to the abdominal cavity and this site is comparatively bloodless during port placement. ${ }^{7}$

The umbilicus was cleaned with spirit soaked cotton gauze and if there was any debris deep inside, was taken out manually. The base of the umbilicus was everted and held with Allis forceps. A vertical incision about one centimeter made with surgical blade no 11, deep inside the umbilicus to hide scar. Deep to the skin tissues, fascia and peritoneum opened and a blunt large 
size artery forceps introduced to widen the opening while maintaining elevation. A trocar was then placed and gas nozzle attached to create pneumoperitoneum. Once trocar is inside the inflated abdominal cavity, telescope was rotated all around 360 degrees to check for any omental or visceral injury.

After concluding the surgery, the surgical wound was irrigated with normal saline solution and skin closed. As there is close proximity of different layers at umbilicus, no additional sutures were made in the subcutaneous fat layer or the skin and we applied a single layer closure.

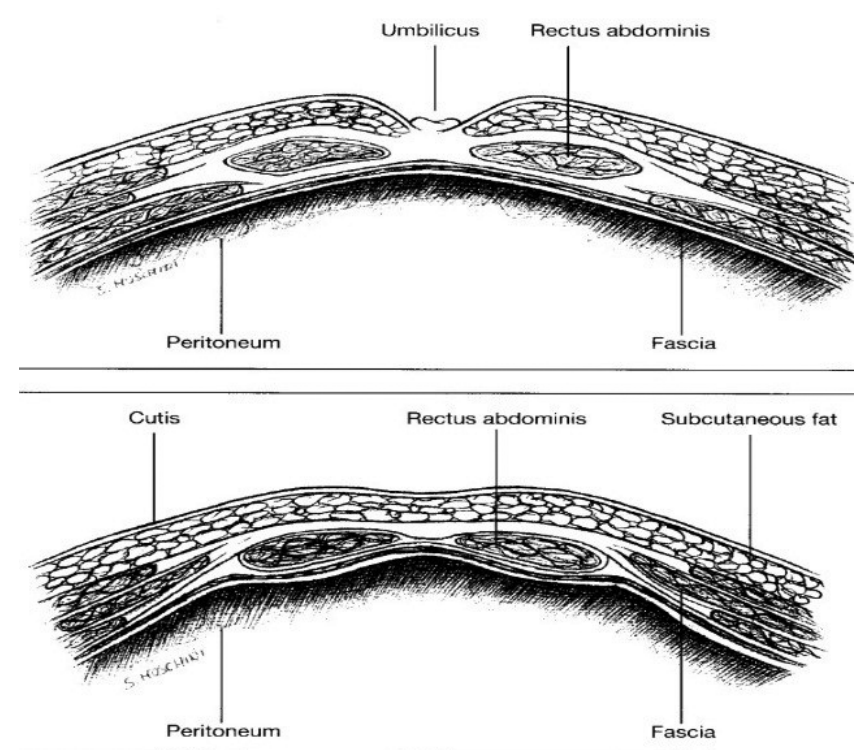

Figure-1. Anatomy of the anterior abdominal wall.

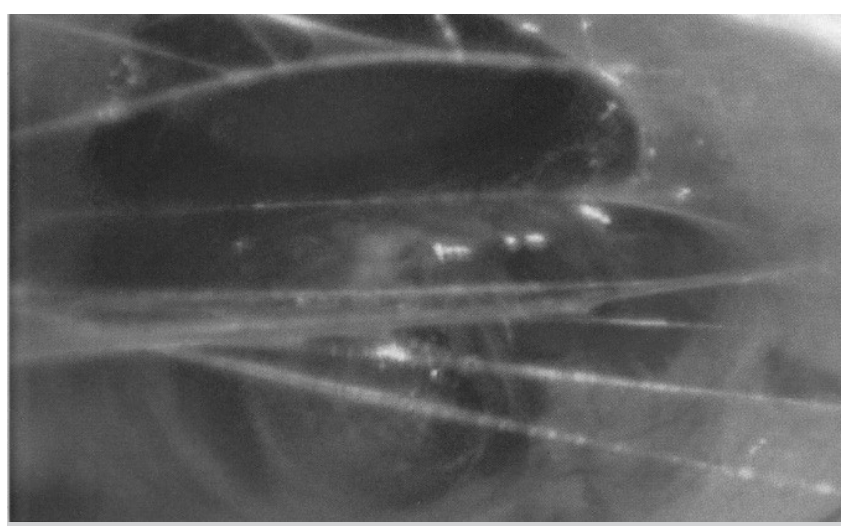

Figure-2. Failed entry for pneumoperitoneum.

\section{RESULTS}

A total number of 238 patients at Unit II of department of General Surgery who underwent different laparoscopic surgeries during this period were included in this study. Patients were allocated randomly into two groups by lottery method; Group A (Trans-Umbilical) and Group B (Infra-Umbilical) 119 and 119 respectively. There were 147 female and 91 male. (M: F ratio of 1 : 1.615).

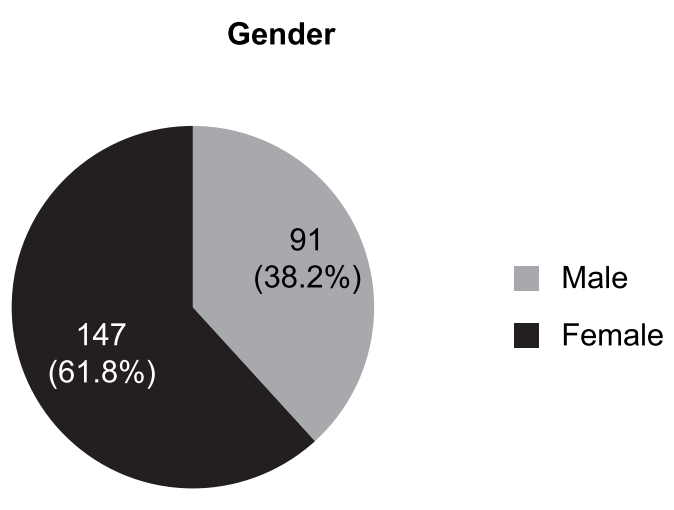

Figure-3. Gender-wise distribution of cases.

The age of the patients ranged from 16 to70 years. The mean age of all patients was 39.7 years. The most, 55 patients were in the age group 31-40 years, the second most common age group was 41-50 years and the least 18 patients were in the age group 61-70 years. Table-I shows age-wise distribution of cases.

\begin{tabular}{|c|c|c|}
\hline Sr. No & \multicolumn{1}{|c|}{ Age Groups } & Frequency (\%) \\
\hline 1 & $16-20$ years & $47(19.74 \%)$ \\
\hline 2 & $21-30$ years & $38(15.94 \%)$ \\
\hline 3 & $31-40$ years & $55(23.1 \%)$ \\
\hline 4 & $41-50$ years & $51(21.41 \%)$ \\
\hline 5 & $51-60$ years & $29(12.18 \%)$ \\
\hline 6 & $61-70$ years & $18(7.56 \%)$ \\
\hline \multicolumn{2}{|c|}{ Table-l. Age group-wise distribution of cases. } \\
\hline
\end{tabular}

The most common laparoscopic surgeries included in this study were laparoscopic cholecystectomy 109 (45.8\%) followed by appendectomy 85 (35.71 \%) and the least commonly performed procedure was diagnostic laparoscopy in 3 patients (1.26\%). Laparoscopic procedures conducted are given in Table-II. 


\begin{tabular}{|c|c|c|}
\hline $\begin{array}{c}\text { Sr. } \\
\text { No }\end{array}$ & Procedure & $\begin{array}{c}\text { Frequency } \\
\text { (\%) }\end{array}$ \\
\hline 1 & Laparoscopic cholecystectomy & $109(45.8 \%)$ \\
\hline 2 & Laparoscopic appendectomy & $85(35.7 \%)$ \\
\hline 3 & Laparoscopic hernia repair & $12(5.04 \%)$ \\
\hline 4 & Ovarian cystectomy/fenestration & $10(4.2 \%)$ \\
\hline 5 & Adhesionolysis & $08(3.3 \%)$ \\
\hline 6 & Orchidopexy & $06(2.5 \%)$ \\
\hline 7 & Varicocelectomy & $05(2.1 \%)$ \\
\hline 8 & Diagnostic laparoscopy & $03(1.3 \%)$ \\
\hline
\end{tabular}

Table-II. Different laparoscopic procedures during the study period.

\section{Complications}

During the surgery and follow up period the complications that occurred in both the groups were recorded. Minor bleeding was seen in 9 TU cases and in 17 cases with IU port. Bleeding stopped automatically.

There was no major vascular injury in any of the patient. We were unable to pass the port in 3 cases of TU site $(2.5 \%)$ the reason being very deep seated umbilical cicatrix, but during placement of port at IU site there was no such failure.

Mean time for port entry (time taken from skin incision to placement of telescope in to the abdominal cavity), in transumbilical was one minute and 22 seconds (range1-5 mints) and the mean time in infraumbilical port was 4 minutes and 35 seconds (range, 2 minutes 40 seconds to 9 mints 10 seconds).
Post-operative recovery means physical activity (mobilize independently walk and going to toilet) was smooth in almost all of the cases. Recovery in trans-umbilical approach was better than that of infra-umbilical route, 10 hours in TU and 17 hours in IU. 107 patients were discharged home on the first post-operative day and rest were kept admitted after 24 hours,(due to pain and delayed gut motility) while in patients where infraumbilical approach was maneuvered 98 patients were discharged on the first post-operative day and rest were kept after 24 hours ( $p)$. The mean hospital stay was 1.1 days (range $0-3$ days).

In Group A, hematoma (diagnosed on the basis of swelling and bluish discoloration) developed in 4 patients and in 7 patients in Group B. Surgical site Infection (parameters were the swelling, the redness, the warmth at the site with or without pus discharge and systemic features like fever and leukocytosis), was seen in 7 patient in Group A $(5.9 \%)$ and 6 patients in Group B (5.0\%). Operative blood loss was minimal in all cases. There were no complications like gas embolism, major vessel injury, bowel injury and death in both groups.

\section{DISCUSSION}

There is no available evidence to support any of the techniques for primary port placement, as per guideline of the European Association of Endoscopic Surgery (EAES).

\begin{tabular}{|c|l|c|c|}
\hline Sr. No & \multicolumn{1}{|c|}{ Complication } & Group A (TU) n (\%) & Group B (IU) n (\%) \\
\hline 1. & Failed pneumoperitoneum & $3(2.5 \%)$ & $0(0 \%)$ \\
\hline 2. & Extra peritoneal insufflation & $2(1.7 \%)$ & $3(2.5 \%)$ \\
\hline 3. & Periumbilical hematoma/bruise & $4(3.4 \%)$ & $7(5.9 \%)$ \\
\hline 4. & Port site Infection(PSI) & $7(5.9 \%)$ & $0(0 \%)$ \\
\hline 5. & Omental injuries & $3(2.5 \%)$ & $17(14.3 \%)$ \\
\hline 6. & Minor vessel injury (including port site bleeding) & $9(7.6 \%)$ & $4(4.2 \%)$ \\
\hline 7. & Port site emphysema & $1(0.8 \%)$ & $5(4.2 \%)$ \\
\hline 8. & Gas leakage & $1(0.8 \%)$ & $3(0.8 \%)$ \\
\hline 9. & Port site omental injury & $3(2.5 \%)$ & $6(5.9 \%)$ \\
\hline 10. & Port site hernia & $1(0.8 \%)$ & $0(0 \%)$ \\
\hline 11. & Postoperative seroma & $3(2.5 \%)$ & $1(0.8 \%)$ \\
\hline 12. & Stitch granuloma & \multicolumn{2}{|c|}{ Table-II. Intra-operative and post-operative complications in TU and IU port placement groups. } \\
& (N = 119 in each group) & \\
\hline
\end{tabular}


There is still controversy regarding the best method for the creation of the pneumoperitoneum. ${ }^{8,9,10}$ There are more than one established ways of putting primary port for pneumoperitoneum, like open Hasson's technique and closed technique, either initially with Veress Needle and then entering trocar or direct trocar entry. ${ }^{2,3,5}$ In this study we used the open technique for placement of primary port at infra-umbilical site.

The commonly used sites for the placement of primary port in laparoscopy are, most of the time infra-umbilical, trans-umbilicus and sometimes supra-umbilical. There has been a continuous effort to minimize the complications along with a better cosmetic effect, as shape of umbilicus has a big impact on the personality of a human body. ${ }^{11}$ About half of the complications related to laparoscopic surgery are due to first port entry (pneumoperitoneum creation) and sometime a life threatening situation arises due to some major vascular injury. ${ }^{11,12}$ That's why creation of the pneumoperitoneum is the first and most critical step of any laparoscopic surgery.

The open technique of placement of primary port was introduced by Hasson, in $1971 .^{3}$ The most important problem encountered was the air leakage in this open technique. This technique, a sort of mini laparotomy consists of an initial incision into the skin and then after dissection and opening the peritoneum allowing direct visualization for the insertion of a blunt trocar, before gas insufflation and laparoscope introduction. ${ }^{13,14}$

This study was carried out to assess and compare the, efficacy and safety of TU port with IU port. In this study the different measured variables were access time, failed entry, minor and major complications, visceral and vascular injury and bleeding, port site infection and length of hospitalization after surgery.

The average/ mean age was 39.7 years. Mean time for portal entry (time taken from skin incision to placement of telescope in to the abdominal cavity), in transumbilical was one minute and 22 seconds (range1-5 mints) and the mean time in infraumbilical port was 4 minutes and 35 seconds (range, 2 minutes 40 seconds to 9 mints 10 seconds) which shows marked difference in both groups. Thus TU appears to be quicker than IU port placement and thus provides a quick, safe, easy to perform and reliable initial access to the peritoneal cavity that is accompanied by excellent functional and cosmetic results.

It has shown a proportional rise in surgical site infection with increase in the size of the port site incision and trocar. Although rare, complications that occur at the port site include bleeding, emphysema, ecchymosis and port site hernia. In a study by Karthik et al., 1.8\% had port site infection followed by bleeding from port site in $0.7 \%$ and omentum-related complications in $0.35 \% .{ }^{15}$ Similar other studies have found no difference in the trans-umbilical and other approaches in terms of complications and patients satisfaction. ${ }^{16}$

In our current study there was no significant difference of wound infection (SSI) in both groups in TU group this was in 7 patients and in IU group this was in 6 patients. Swab culture was taken and the patients were managed accordingly. The patients to have SSI must have localized erythema, edema, and hotness, accompanied by subjective pain. The wound may be with or without purulent discharge. Thus TU appears to be similar to other approaches in terms of postoperative complications.

The appearance of the umbilicus is an utmost important part of one's personality and partner's attractiveness. Post-operative cosmesis and development of hernia must therefore be under consideration while selecting port insertion technique. In our study one patient in TU group and 3 in IU group developed incisional hernia, labeled on the finding of protrusion or bulge present on cough impulse. A study by Senturk et al., compared Cosmetic results in transumbilical, infraumbilical and supraumbilical techniques. They compared score of the Vancouver scar scale and observed significantly better cosmesis in patients who had transverse incisions. ${ }^{17} \mathrm{TU}$ technique thus appears to have better cosmesis 
and reduces the chances of post-operative hernia formation rates as compared to IU technique. Overall it appears that TU approach has obvious benefits over IU approach in view of safety, speed, accuracy, per operative/ postoperative complications and cosmetic outcome.

\section{CONCLUSION}

In the study, it was found that the trans-umbilical approach for creation of pneumoperitoneum had statistically better results than the infra-umbilical approach, in terms of the time required for the successful start of creation of pneumoperitoneum, post-operative complications and better cosmetic result. Therefore trans-umbilical approach is an effective alternative to infra-umbilical approach for creation of pneumoperitoneum. Infra-umbilical approach, in fact is a sort of mini laparotomy rather than a small opening deep inside the umbilicus. If strict aseptic techniques are observed there is no increased risk of port site infection in transumbilical approach.

\section{Copyright@ 15 Dec, 2019.}

\section{REFERENCES}

1. Resutra R, Mahajan N, Gupta R. Trans umbilical first trocar access during laparoscopic surgery. International Journal of Research in Medical Sciences. 2019 Aug;7(8):3039.

2. Ogaick M, Martel G. Advances in abdominal access for laparoscopic surgery: A review. Open Access Surgery. 2014; 7:81.

3. Chotai NR, Choksi BB, Damor S, Bhedi A. Intraperitoneal access by closed method (veress needle) versus open (Hasson's) method in laparoscopic surgery to create pneumoperitoneum. International Surgery Journal. 2017 Jul 24; 4(8):2786-90.

4. Ravindranath GG, Reddy SR. Laparoscopic port site complications: A study in a tertiary care centre. International Surgery Journal. 2016 Dec 9; 3(3):1121-4.

5. Rajkhowa K, Gogoi M, Baruah I. Periumbilical Incision versus Intraumbilical Incision for Laparoscopic Appendectomy: A randomized comparative study. International Journal of Scientific Study. 2016 Nov 1; $4(8): 172-5$.
6. RANZCOG. Use of the Veress needle to obtain pneumoperitoneum prior to laparoscopy. Consensus statement of the Royal Australian \& New Zealand College of Obstetricians \& Gynecologists (RANZCOG) and the Australasian Gynecological Endoscopy and Surgery Society (AGES). July 2017.

7. Alkatout I, Mettler L, Maass N, Noé GK, Elessawy M. Abdominal anatomy in the context of port placement and trocars. Journal of the Turkish German Gynecological Association. 2015; 16(4):241.

8. Panigrahi SK, Behera CR, Pujari PS, Mishra A, Kanungo $A$, Analysis of port site complications in laparoscopic surgeries in a tertiary care centre. International Journal of Scientific Research. 2018:7(1):241-43.

9. Tariq M, Ahmed R, Rehman S, Sajjad M. Comparison of direct trocar insertion with other techniques for laparoscopy. Journal of the College of Physicians and Surgeons--Pakistan: JCPSP. 2016 Nov 1; 26(11):917-9.

10. Roy AN, Ghatak S. A simple and safe technique of creating closed pneumoperitoneum. Indian Journal of Surgery. 2013 Aug 1; 75(4):262.

11. Aslam R, Kalim M, Shamsi H, Gul A, Aman Z. Frequency of common complications of veress needle used for creating pneumoperitoneum in laparoscopic cholecystectomy. KJMS. 2018 Sep; 11(3):435.

12. Krishnakumar S, Tambe P. Entry complications in laparoscopic surgery. Journal of gynecological endoscopy and surgery. 2009 Jan; 1(1):4.

13. Toro A, Mannino M, Cappello G, Di Stefano A, Di Carlo I. Comparison of two entry methods for laparoscopic port entry: technical point of view. Diagnostic and therapeutic endoscopy. 2012;2012.

14. Ogaick M, Martel G. Advances in abdominal access for laparoscopic surgery: A review. Open Access Surgery. 2014; 7:81.

15. Karthik S, Augustine AJ, Shibumon MM, Pai MV. Analysis of laparoscopic port site complications: A descriptive study. Journal of minimal access surgery. 2013 Apr; 9(2):59.

16. Bouffard-Cloutier A, Paré A, McFadden N. Periumbilical vs transumbilical laparoscopic incision: A patients' satisfaction-centered randomised trial. International Journal of Surgery. 2017 Jul 1; 43:86-91.

17. Şentürk MB, Doğan O, Polat $M$, Kılıçcı Ç, Pulatoğlu Ç, Tayyar AT. Cosmetic outcomes of infraumbilical, supraumbilical, and transumbilical entry routes in laparoscopic surgery. Turkish journal of surgery. 2018; 34(4):290. 


\title{
We cannot solve our problems with the same thinking we used when we created them.
}

\author{
"Albert Einstien"
}

\begin{tabular}{|c|c|c|c|}
\hline \multicolumn{4}{|c|}{ AUTHORSHIP AND CONTRIBUTION DECLARATION } \\
\hline Sr. \# & Author(s) Full Name & Contribution to the paper & Author(s) Signature \\
\hline 1 & Waseem Sadiq Awan & Concept \& Study design. & \\
\hline 2 & Raza Farrukh & Data collection \& Critically & \\
\hline 3 & Umme Balqees & Data collection. & \\
\hline 4 & Ahmed Hassan Khan & Concept \& Data collection. & \\
\hline 5 & Ahmed Aziz Jillani & Data collection \& Statistical & \\
\hline 6 & & Data collection \& Drafting. & \\
\hline
\end{tabular}

